\title{
Field-Free Isolation of Exosomes from Extracellular Vesicles by Microfluidic Viscoelastic Flows
}

\author{
Chao Liu, ${ }^{\ddagger}$ Jiayi Guo, ${ }^{\ddagger}, \|$ Fei Tian, ${ }^{\ddagger}$ Na Yang, Fusheng Yan, ${ }^{\ddagger}$ Yanping Ding, JingYan Wei, ${ }^{\ddagger}$ \\ Guoqing $\mathrm{Hu}, *,+\S$ Guangjun Nie, ${ }^{*},+, \S_{0}$ and Jiashu Sun ${ }^{*},+, \S_{0}$
}

${ }^{\dagger}$ State Key Laboratory of Nonlinear Mechanics, Institute of Mechanics, Chinese Academy of Sciences, Beijing 100190, China

${ }^{\ddagger}$ CAS Key Laboratory of Standardization and Measurement for Nanotechnology, CAS Key Laboratory for Biomedical Effects of Nanomaterials and Nanosafety, CAS Center for Excellence in Nanoscience, National Center for Nanoscience and Technology, Beijing 100190, China

${ }^{\S}$ University of Chinese Academy of Sciences, Beijing 100049, China

"College of Pharmaceutical Science, Jilin University, Changchun 130021, China

\section{Supporting Information}

\begin{abstract}
Exosomes, molecular cargos secreted by almost all mammalian cells, are considered as promising biomarkers to identify many diseases including cancers. However, the small size of exosomes $(30-200 \mathrm{~nm})$ poses serious challenges in their isolation from complex media containing a variety of extracellular vesicles (EVs) of different sizes, especially in small sample volumes. Here we present a viscoelasticitybased microfluidic system to directly separate exosomes from cell culture media or serum in a continuous, size-dependent, and label-free manner. Using a small amount of biocompatible polymer as the additive in the media to control the viscoelastic forces exerted on EVs, we are able to achieve a high separation purity $(>90 \%)$ and recovery $(>80 \%)$ of exosomes. The proposed technique may serve as a versatile platform to facilitate exosome analyses in diverse biochemical applications.
\end{abstract}

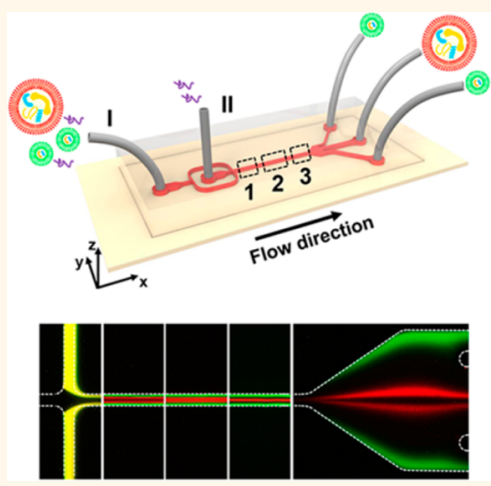

KEYWORDS: exosomes, microvesicles, separation, viscoelasticity, microfluidics

$\mathrm{E}$ xosomes have recently gained huge interest in the scientific community as potential diagnostic biomarkers and therapeutic vehicles for diverse diseases, including cancer, infectious diseases, and neurodegenerative disorders. ${ }^{1-4}$ Exosomes (from 30 to $200 \mathrm{~nm}$ in diameter $^{1}$ ) are small extracellular vesicles (EVs) containing proteins and nucleic acids from their originating cells, playing vital roles in intercellular communication., Although exosomes are abundant in body fluids such as blood, urine, synovial fluid, and saliva, the copresence of several other types of EVs in biofluids often affects the accurate analysis of exosomes. To better interpret the information carried by exosomes, there is an urgent need to isolate and purify the exosomes from a variety of EVs. However, the small size of exosomes and the complex nature of biofluids pose significant challenges to their separation. Conventional batch techniques for isolation of exosomes include ultracentrifugation, density-based separation, and immune-affinity capture of exosomes, based upon their size, floatation density, and marker proteins present on the membrane, respectively. These strategies often require large sample volumes, cumbersome operations, and expensive instruments, ${ }^{7,8}$ which can lead to low recovery, contamination, and high cost. ${ }^{9}$

Viscoelastic microfluidics is a simple and label-free technique for particle manipulation, ${ }^{10-14}$ relying on the particle migration caused by size-dependent elastic lift forces in a viscoelastic medium. Viscoelastic microfluidics has demonstrated itself as an efficient technology to separate tumor cells, ${ }^{15}$ blood cells, ${ }^{16,17}$ bacteria, ${ }^{15}$ droplets, ${ }^{18}$ and microspheres. ${ }^{11,19-21}$ In comparison with other microfluidic separators resorting to acoustics, ${ }^{22-24}$ electrophoresis, $^{25}$ magnetics, ${ }^{26,27}$ and so forth, ${ }^{28,29}$ the viscoelastic manipulation of particles can be continuously performed without any externally applied fields, dramatically simplifying the design and fabrication of microfluidic devices. Most importantly, viscoelastic microfluidics enables the precise manipulation of submicrometer particles in small sample volumes, which is difficult to achieve by inertial microfluidics. $^{30,31}$ Despite these aforementioned advantages, the

Received: April 1, 2017

Accepted: July 5, 2017

Published: July 5, 2017 
viscoelastic separation by microfluidics has not been translated to sort exosomes and other EVs, due to the insufficient elastic lift forces acting on nanoscale particles.

In this work, we present a viscoelastic microfluidic system for size-dependent and field-free separation of exosomes from other large EVs. EVs are laterally driven and subsequently fractionated according to their sizes in a diluted poly(oxyethylene) (PEO) solution. Using analytical model and numerical simulation, the system is first optimized in terms of microchannel geometry, medium elasticity, and flow conditions. Under the optimal conditions with a size cutoff of $200 \mathrm{~nm}$, the exosomes are continuously separated from large EVs from cell culture medium or serum. The presented system shows great potential as a versatile and label-free separation tool for exosome analyses in applications of biology, chemistry, and nanotechnology.

\section{RESULTS}

Design Principle of Viscoelastic Separation in Microfluidics. A microfluidic device consisting of a high-aspect-ratio (AR, defined as the ratio of the microchannel height $H$ to the width $W)$ straight microchannel $(H=50 \mu \mathrm{m}$ and $W=20 \mu \mathrm{m})$, two inlets, and three outlets is designed for viscoelastic separation (Figure 1A and Figure S1 for the details on the microchannel design). The microfluidic device is assembled by bonding a poly(dimethylsiloxane) (PDMS) layer containing the

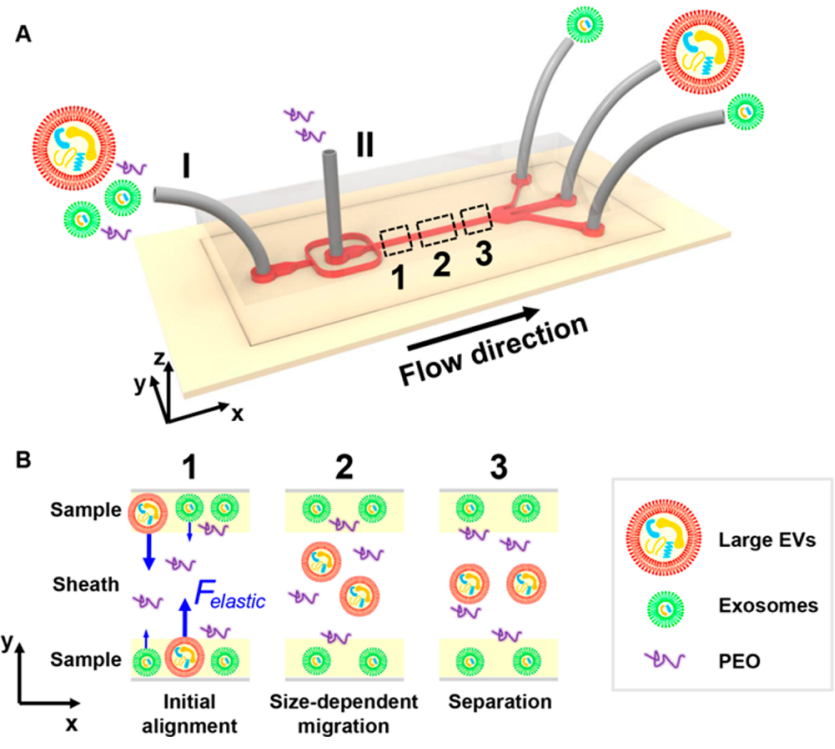

Figure 1. (A) Schematic of the microfluidic chip for exosome separation from large EVs. The microfluidic chip consists of a highaspect-ratio straight microchannel ( $50 \mu \mathrm{m}$ high and $20 \mu \mathrm{m}$ wide), two inlets, and three outlets. Sample and sheath fluids containing a low concentration of PEO are introduced into the microchannel from inlet I and inlet II, respectively. After separation, exosomes are collected at the two side outlets, and large EVs are collected at the middle outlet. (B) Schematic illustration of the separation mechanism in viscoelastic microfluidics (not to scale). The sample fluid is initially aligned along the sidewalls by the sheath fluid. The elastic lift forces (blue arrows) arising from fluid viscoelasticity (due to the presence of PEO) drive exosomes and large EVs toward the microchannel centerline at a size-dependent rate. Large EVs finally reach the microchannel centerline, whereas exosomes remain near the microchannel sidewalls in the downstream microchannel, resulting in their size-dependent separation. embedded microchannel with a glass substrate. Inlet I and inlet II are used for the introduction of sample and sheath fluids containing a low concentration of PEO $(0.1 \%)$, respectively. The addition of PEO makes the fluids highly viscoelastic and consequently generates elastic lift forces on suspended nanoparticles in a Poiseuille flow to control their lateral positions. The suspended nanoparticles with different diameters (a) from inlet I are first aligned around the microchannel sidewalls, by controlling the flow rate ratio of inlet II to inlet I and laterally driven toward the microchannel centerline with size-dependent lateral speeds controlled by the elastic lift forces $\left(\sim a^{3}\right)$. Large nanoparticles that migrate faster to the microchannel centerline are collected from the middle outlet, whereas small nanoparticles with lower migration speed are collected from two-sided outlets (Figure S2 for the flow field at the outlet). In this manner, a label-free and continuous separation of nanoparticles of different sizes is achieved (Figure 1B).

Theoretical Prediction. To predict the migration of nanoparticles in viscoelastic microfluidics, we develop an analytical model to characterize the particle dynamics. This analytical model is constructed by considering the elastic lift forces, the inertial lift forces, and the viscous drag forces. In a Poiseuille flow of the PEO solution (Figure 2A,B), the elastic
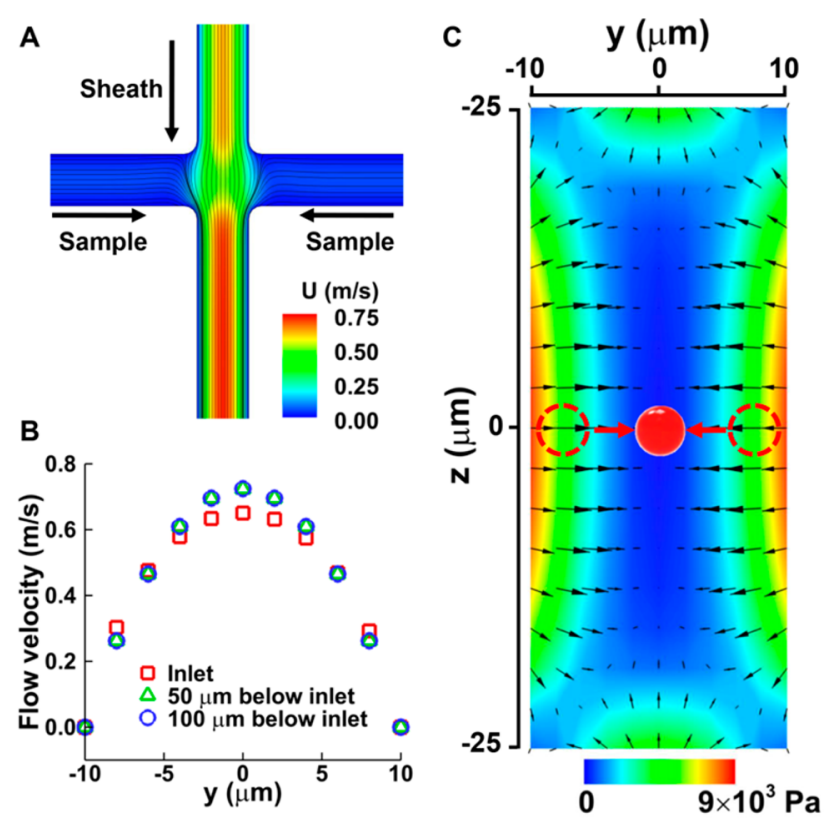

Figure 2. (A) Flow field at the inlets showing that the sample fluid is initially aligned into two narrow side streams by the sheath fluid. (B) Velocity distributions showing that the Poiseuille flow becomes fully developed within the $100 \mu \mathrm{m}$ microchannel length. (C) The elastic lift forces (black arrows) arise from the nonuniform first normal stress difference $\left(N_{1}\right.$, contour plot) and direct down the gradient of $N_{1}$. The elastic lift forces predominately drive nanoparticles toward the microchannel centerline (red arrows). The conditions are $c=0.1 \mathrm{wt} \%$ and $Q=1400 \mu \mathrm{L} / \mathrm{h}$.

lift forces arise from the imbalance of first normal stress difference $\left(N_{1}\right)$ and drive particles toward the microchannel centerline (Figure 2C). The elastic lift forces are proportional to the variation of normal stress $N_{1}$ over the particle volume, i.e., $F_{\mathrm{e}}=C_{\mathrm{e}} a^{3} \nabla N_{1}$, where $C_{\mathrm{e}}$ is the elastic lift coefficient. The inertial lift forces arising from the nonlinear effects at finite Reynolds number $\left(R e=\rho U_{\max } W / \eta\right.$, where $\rho$ is the medium 
density, $U_{\max }$ is the maximum flow velocity, and $\eta$ is the medium viscosity) are expressed as $F_{\mathrm{i}}=C_{\mathrm{i}} \rho U_{\max }^{2} a^{4} / W^{2}$, where $C_{\mathrm{i}}$ is the inertial lift coefficient. ${ }^{32,33}$ The viscous drag forces are expressed as $F_{D}=3 \pi a \eta V$ according to the Stokes' law, where $V$ is the lateral migration speed.

The trajectories of nanoparticles inside the microchannel are predicted based on the competition between $F_{\mathrm{e}}$ and the $F_{\mathrm{D}}$. The shear viscosities of PEO solutions at low concentrations ( $c$ $=0.025-0.15 \mathrm{wt} \%$ in the present study) are nearly constant irrespective of shear rate (Figure 3A). The used PEO solution is
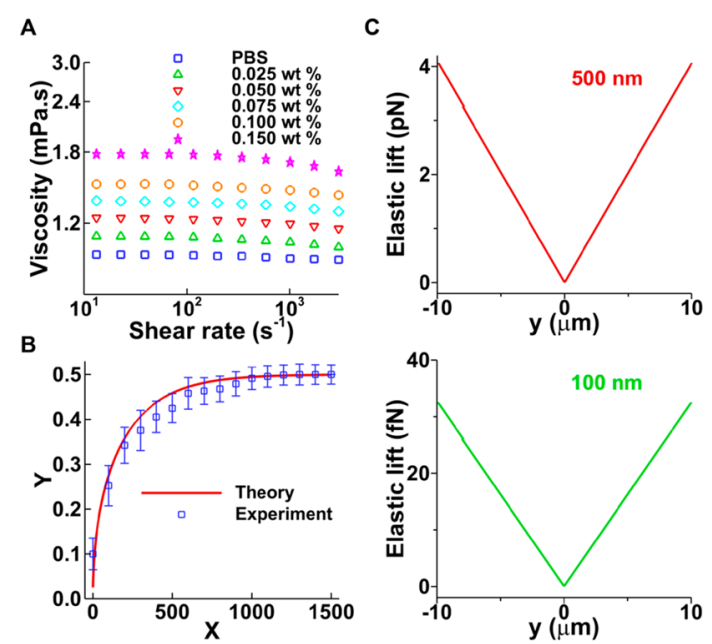

Figure 3. (A) Shear viscosities of solutions at different PEO concentrations. The viscosities of the solutions are measured at shear rates of $10-3000 \mathrm{~s}^{-1}$. The measurements are conducted at 20 ${ }^{\circ} \mathrm{C}$ with a cone and plate geometry $(50 \mathrm{~mm}, 0.017 \mathrm{rad})$ installed on a rotational rheometer (Physica MCR301, Anton PaarGmBH, Germany). (B) Determination of the elastic lift coefficient as 0.0184 by fitting analysis (red line) of the experimental observation (blue markers) of $a=500 \mathrm{~nm}, c=0.1 \mathrm{wt} \%$, and $Q=1400 \mu \mathrm{L} / \mathrm{h}$. Error bars are calculated from three repeat measurements. (C) Elastic lift forces on nanoparticles with diameters of 500 and 100 $\mathrm{nm}$ along the short main axis (width) of the microchannel crosssection for $c=0.1$ wt $\%$ and $Q=1400 \mu \mathrm{L} / \mathrm{h}$.

thus considered as an Oldroyd-B fluid, whose constitutive equation can be described by the Oldroyd-B model. For the approximate planar Poiseuille profile in the high AR microchannel, ${ }^{10,34}$ we calculate the particle trajectory based on the force balance along the width:

$$
\begin{aligned}
\frac{\mathrm{d} Y}{\mathrm{~d} X}+\frac{(1-2 Y) \Phi}{\left(Y-Y^{2}\right)}+\frac{\Theta}{\left(Y-Y^{2}\right)}=0, \Phi=\frac{8 C_{\mathrm{e}} \kappa^{2} W i}{3 \pi}, \\
\Theta=\frac{C_{\mathrm{i}} \kappa^{3} R e}{12 \pi}
\end{aligned}
$$

where $X$ and $Y$ are dimensionless positions (normalized by $W$ ) along the streamwise and width directions, respectively; $\kappa$ is the blockage ratio of the nanoparticle $(a / W)$; and the Weissenberg number $(W i)$ is the ratio of $N_{1}$ to the viscous stress $\dot{\gamma} \eta$, which can be expressed as $W i=2 \lambda \eta_{\mathrm{p}} \dot{\gamma} / \eta$ based on the Oldroyd-B model $\left(N_{1}=2 \eta_{\mathrm{p}} \lambda \dot{\gamma}^{2}\right) .{ }^{35}$ Here $\eta_{\mathrm{p}}$ is the PEO contribution of the solution viscosity $\left(\eta=\eta_{\mathrm{s}}+\eta_{\mathrm{p}}\right.$, where $\eta_{\mathrm{s}}$ is the solvent viscosity) and $\lambda$ is the relaxation time of the PEO solution. We determine $C_{\mathrm{e}}$ as $1.84 \times 10^{-2}$ by fitting the experimental observation under the conditions of $a=500 \mathrm{~nm}, c=0.1 \mathrm{wt} \%$, and $Q=1400 \mu \mathrm{L} / \mathrm{h}$ (Figure $3 \mathrm{~B}$ ) and thus are able to calculate the elastic lift forces: the above conditions could provide sufficient elastic lift forces
(4.1 pN) on $500 \mathrm{~nm}$ particles while keeping $100 \mathrm{~nm}$ particles (only $33 \mathrm{fN}$ ) around the sidewalls of microchannels (Figure 3C). The $C_{i}$ is determined from the perturbation method by Schonberg and Hinch, ${ }^{36}$ which is applicable to the conditions of $\kappa \ll 1$ ( $\kappa=0.025$ and 0.005 for 500 and $100 \mathrm{~nm}$ particles, respectively) and $\operatorname{Re}=1-15$ ( $R e=10$ for $c=0.1 \mathrm{wt} \%, Q=$ $1400 \mu \mathrm{L} / \mathrm{h}$ ). Compared to the elastic lift forces, the inertial lift forces are at least 1 order of magnitude weaker over the entire range of lateral positions (Figure S3), which is consistent with the observation in a Newtonian medium that the $500 \mathrm{~nm}$ particles are only slightly lifted away from the sidewalls and cannot be separated from $100 \mathrm{~nm}$ particles under pure inertial effects (Figure S4). Therefore, the elastic lift forces are the dominant driving forces for the lateral migration and the effective separation of nanoparticles.

The particle trajectories under different conditions are obtained by numerically solving eq 1 for the corresponding $\Phi$ that includes the effects of particle size and fluid viscoelasticity. For PEO solutions of low concentrations $(c<$ 0.19 wt $\%$, the overlapping concentration for PEO of 600 $\left.\mathrm{kDa}^{13}\right), \eta_{\mathrm{p}}$ and $\lambda$ can be expressed as $0.072 \eta_{\mathrm{s}} c M_{\mathrm{w}}{ }^{0.65}$ and 0.128 $M_{\mathrm{w}}^{2.07} \eta_{\mathrm{s}} c^{0.65} / N_{\mathrm{A}} k_{\mathrm{B}} T{ }^{37-39}$ respectively. Therefore, the dimensionless parameter $\Phi$ scales as $a^{2} c^{1.65} U$, indicating that the particle trajectory is predominately dependent on the particle size, PEO concentration, and flow speed. Under the following conditions $(c=0.1$ wt $\%, Q=1400 \mu \mathrm{L} / \mathrm{h})$, the trajectories of nanoparticles of different sizes $(a=1000,500,200$, and 100 $\mathrm{nm})$ are shown in Figure 4A. All the nanoparticles starting from

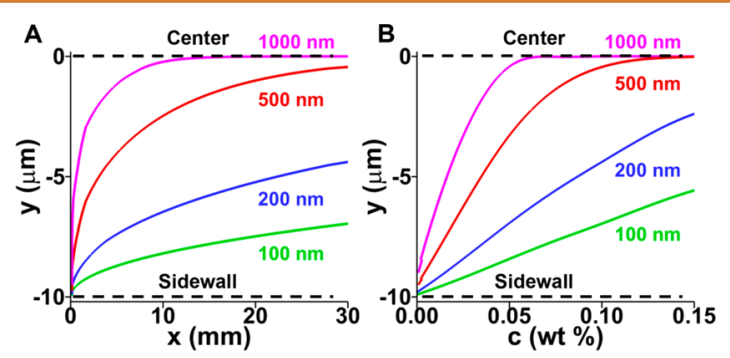

Figure 4. (A) Calculated trajectories of nanoparticles with different diameters of $1000,500,200$, and $100 \mathrm{~nm}$ along the flow direction. (B) Calculated lateral positions of 1000, 500, 200, and $100 \mathrm{~nm}$ particles at the end of the microchannel at various PEO concentrations ranging from 0 to 0.15 wt \%.

the sidewall migrate toward the microchannel centerline only with a difference in migration speed $\left(\Phi \approx a^{2}\right)$. Within the microchannel length of $30 \mathrm{~mm}$, large particles $(a=1000$ and $500 \mathrm{~nm}$ ) reach the microchannel centerline, whereas small particles $(a=200$ and $100 \mathrm{~nm})$ still remain near the microchannel sidewalls, eventually achieving a size-dependent separation. We further calculate the lateral positions of particles at the end of the microchannel in PEO solutions of $c$ ranging from 0 (deionized water) to $0.15 \mathrm{wt} \%$ (Figure 4B). The migration behaviors of particles are sensitive to the PEO concentration: $500 \mathrm{~nm}$ particles can be driven toward the microchannel centerline at a low $c$ of 0.1 wt \% (Figure S5). The lateral migration distance monotonically increases with $c$ for all investigated $a$. Increasing $c$ beyond the value at which large particles reach the centerline can reduce the separation distance, and thus there is a window of $c$ for the best separation performance. For the nanoparticles of $a=500$ and $100 \mathrm{~nm}$, the largest separation distance is obtained at $c=0.1 \mathrm{wt} \%(c=0.075$ wt $\%$ for $a=1000$ and $100 \mathrm{~nm}$; Figure S6). According to $\Phi \approx$ 
$U$ (compared with $\Phi \approx a^{2} c^{1.65}$ ), the particle positions are less sensitive to the flow speed within a certain range, which is validated by experimental results of $500 \mathrm{~nm}$ particles in a $0.1 \mathrm{wt}$ $\%$ PEO solution (Figure S7). We choose the flow rate of 1400 $\mu \mathrm{L} / \mathrm{h}$ for the following nanoparticle separation considering that the particle focusing gets worse at a flow speed higher than 0.7 $\mathrm{m} / \mathrm{s}(Q=1400 \mu \mathrm{L} / \mathrm{h})$ (Figure S7).

Validation Using PS Particles. The separation performance is first validated using polystyrene (PS) particles. Samples are prepared by mixing fluorescent PS particles of $a=500 \mathrm{~nm}$ (red color) and $100 \mathrm{~nm}$ (green color) with the number ratio of 1:2.5 (volume ratio of 50:1). The sample and sheath flow rates are set to 200 and $1200 \mu \mathrm{L} / \mathrm{h}$, respectively. Both the sample and sheath fluids are prepared in a phosphate-buffered saline (PBS) solution containing 0.1 wt \% PEO. The fluorescent images show a size-dependent separation of PS particles in the microchannel (Figure 5A, left). The $500 \mathrm{~nm}$ particles reach the

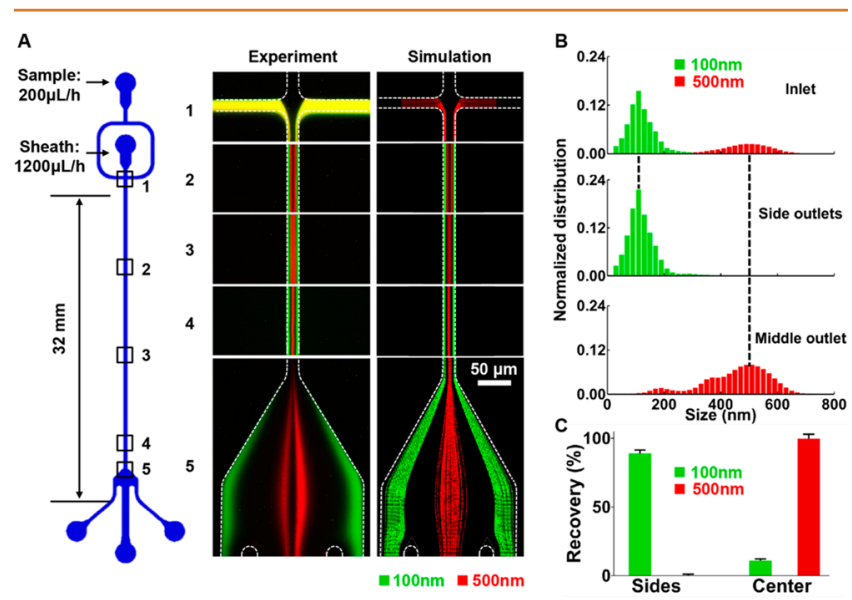

Figure 5. Separation of the binary mixture of $100 \mathrm{~nm}$ (green) and $500 \mathrm{~nm}$ (red) PS particles. (A) Particle trajectories from the experiment (left) and the simulation (right) at $c=0.1 \mathrm{wt} \%$ and $Q=$ $1400 \mu \mathrm{L} / \mathrm{h}$ are in good agreement, while the large and small nanoparticles are collected at the center and side outlets, respectively. (B) Particle size distributions measured by NTA confirm a good performance of size-dependent separation of nanoparticles. (C) Analysis based on NTA data shows the high recovery rates (>90\%) for both 100 and $500 \mathrm{~nm}$ nanoparticles.

centerline within a $30 \mathrm{~mm}$ microchannel length and exit through the center outlet, whereas the $100 \mathrm{~nm}$ particles remain near the sidewalls and exit through the side outlets. The numerically calculated trajectories obtained using Lagrangian tracking simulation agree well with the fluorescent observation (Figure 5A, right), showing a complete separation. The separation performance is further confirmed by nanoparticle tracking analysis (NTA) measurement of the size distribution of particles (Figure 5B). The initial mixture has two distinct particle populations with median diameters at 110 and $490 \mathrm{~nm}$, respectively. After the viscoelastic separation, the large and small particles are separately collected at the center and side outlets, respectively. The separation efficiencies of these two particle types are determined to be above $90 \%$ from the NTA data (Figure 5C). We further investigate the separation performance at different particle concentrations. The concentration of $100 \mathrm{~nm}$ particles is prepared at $0.0002-0.02 \mathrm{wt} \%$ with a constant concentration of 0.01 wt $\%$ for $500 \mathrm{~nm}$ particles. The number ratio of 100 to $500 \mathrm{~nm}$ particles ranges from 2.5 to 250 . The recovery rate remains above $80 \%$ over the entire concentration range (Figure S8).

Exosome Separation. We further use the present technique to separate exosomes from large EVs. The size of exosomes ranges from 30 to $200 \mathrm{~nm}$ with an approximate median value of $100 \mathrm{~nm},{ }^{1}$ whereas the size of large EVs ranges from 200 to $1000 \mathrm{~nm}^{40,41}$ Assuming that the migration behaviors of exosomes and large EVs are similar to those of 100 and $500 \mathrm{~nm}$ nanoparticles, we use the operation conditions for the binary mixture of 100 and $500 \mathrm{~nm}$ PS particles to separate exosomes from large EVs. The mixtures of exosomes and large EVs are obtained from cell culture media by ultracentrifugation and filtration. Before separation, the mixture is characterized by NTA measurement, which shows two distinct particle populations with median diameters at $130 \mathrm{~nm}$ (corresponding to exosomes) and $600 \mathrm{~nm}$ (corresponding to large EVs), respectively. After viscoelastic separation, the size distributions of EVs collected from the middle and side outlets are also measured by NTA (Figure 6A). The recovery rate and purity of exosomes (defined by size $<200 \mathrm{~nm}$ ) are determined to be $80 \%$ and $94 \%$ from the NTA data, respectively. We next use atomic force microscopy (AFM) to observe the mixture of exosomes and large EVs before and after separation. The AFM image shows the presence of both large and small EVs before separation, but only small exosomes are observed in the AFM image of samples collected from the side outlet after separation, indicating a good separation performance (Figure 6B). Moreover, for fluorescent observation, we separately label the exosomes with PKH26 dye (green color) and large EVs with PKH67 dye (red color) before separation. Fluorescent microscopy of EVs at the inlet shows the coexistence of both green and red dots, indicating a mixture sample of exosomes and large EVs. In comparison, fluorescent images of samples collected at the middle and side outlets show the predominance of red (corresponding to large EVs) and green (corresponding to exosomes) dots, respectively (Figure 6C). These experimental results demonstrate the label-free and high-efficient separation of exosomes from large EVs in the present microfluidic device.

We further apply the present technique to isolate exosomes from fetal bovine serum (FBS, Wisent Inc., Canada) without any presample preparation to demonstrate its feasibility for realworld applications. The untreated serum samples are processed in 0.1 wt \% PEO at sheath/sample flow rates of $1200 / 200 \mu \mathrm{L} /$ h. The characterization of initial samples by NTA measurement shows two mode diameters at $100 \mathrm{~nm}$ (corresponding to exosomes) and $400 \mathrm{~nm}$ (corresponding to large EVs), respectively (Figure $7 \mathrm{~A}$ ). After viscoelastic separation, the samples collected from the side and center outlets are also characterized by NTA, showing two distinct particle populations with a size cutoff of $200 \mathrm{~nm}$. The recovery rate and purity of exosomes $(<200 \mathrm{~nm})$ are determined to be $93.6 \%$ and $96 \%$ based on the NTA data, respectively (Figure 7B), showing a high-efficient separation of exosomes from serum.

Comparison of Varying Techniques for Exosome Separation. In comparison with conventional exosome separation techniques (Table S1), the present method is able to obtain comparable recovery and purity of exosomes with easy operation and low cost. Specifically, the viscoelastic separation in this work has a much higher recovery $(>80 \%)$ of exosomes than that of ultracentrifugation $(5-25 \%)$, which is one of the most accessible batch techniques for exosome isolation. ${ }^{42}$ The remarkable merit of this microfluidic system 

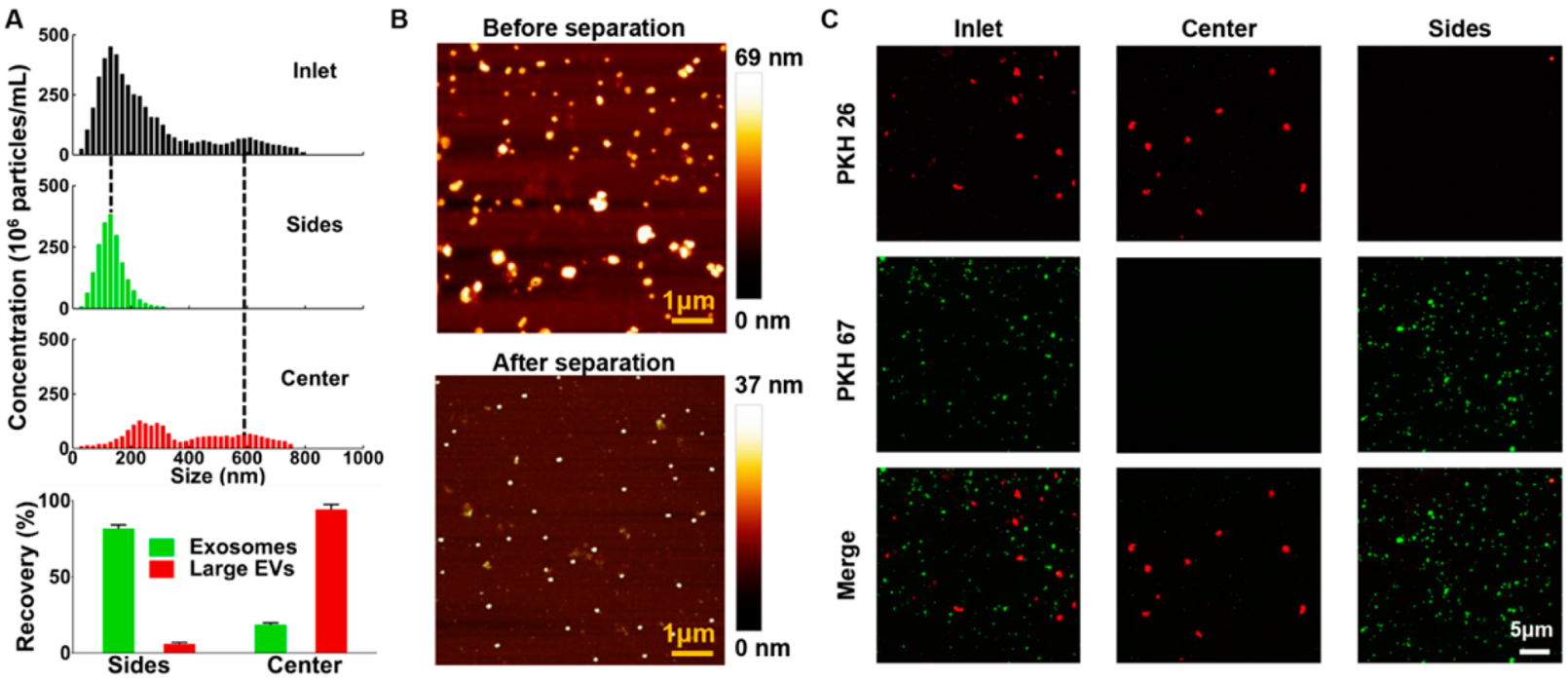

Figure 6. Characterization of separation of exosomes from large EVs. (A) NTA measurement of the size distribution of the mixture of exosomes and large EVs before separation and that of EVs collected from different outlets (top). Recovery rates of exosomes and large EVs calculated based on NTA data (bottom). (B) AFM image of the mixture of exosomes and large EVs before separation (top) and AFM image of EVs collected from the side outlet after separation (bottom). (C) Fluorescent imaging of exosomes (prelabeled with PKH26 dye, green color) and large EVs (prelabeled with PKH67 dye, red color) from the inlet (before separation) and different outlets (after separation), indicating a good separation between exosomes and large EVs.

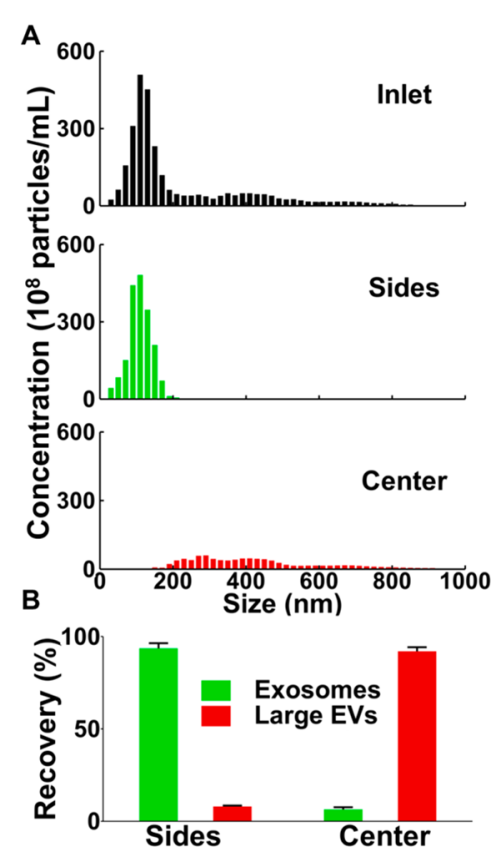

Figure 7. Separation of exosomes and large EVs in fetal bovine serum (FBS) without any presample preparation. (A) NTA measurement of the size distribution of EVs in FBS before separation and that of EVs collected from different outlets. (B) Recovery rates of exosomes and large EVs determined based on NTA data.

lies in its speed, low cost, and ready accommodation for handling the small volume of EVs $(\sim 100 \mu \mathrm{L})$. The passage time of exosomes through the microchannel is less than $0.1 \mathrm{~s}$ (vs 2$5 \mathrm{~h}$ for ultracentrifugation ${ }^{28}$ ), minimizing their physical damage during the process (Figure S9). Comparing with nanoscale deterministic lateral displacement (DLD), another lab-on-achip system for field-free and label-free exosome separation, ${ }^{43}$ our system allows for a much higher processing speed $(200 \mu \mathrm{L} /$ $\mathrm{h}$ in our work $v s 12 \mathrm{~nL} / \mathrm{h}$ in nano DLD), as well as the use of a

much larger channel structure $(20 \mu \mathrm{m}$ in our work $v s 235 \mathrm{~nm}$ in nano DLD) to minimize the risk of clogging. The present sample throughput is still limited compared to conventional macroscale separation methods, such as ultracentrifugation and membrane filtration. However, its handling capability could be largely enhanced by parallelization or using a slit-shaped microchannel design, with an improved sample throughput up to 3 orders of magnitude in particle separation. ${ }^{44,45}$

\section{CONCLUSIONS}

In this work, we have developed a viscoelasticity-based microfluidic system for field-free, label-free, and continuous separation of exosomes from large EVs based on their sizes. A high separation purity $(>90 \%)$ and recovery $(>80 \%)$ of exosomes are achieved by adding a small amount of PEO $(0.1 \mathrm{wt} \%)$ into the cell culture medium or pure serum, without any complex or time-consuming operations. The size cutoff in viscoelasticity-based microfluidics can be easily controlled using different PEO concentrations. On the basis of this sizedependent viscoelastic separation strategy, we envision the handling of diverse nanoscale objects, such as gold nanoparticles, DNA origami structures, and quantum dots.

\section{MATERIALS AND METHODS}

Device Design and Fabrication. The microchannel consists of two inlets for sheath and sample fluids, respectively, a straight separation section with a width $(W)$ of $20 \mu \mathrm{m}$ and a length $(L)$ of 30 $\mathrm{mm}$, and three outlets for exosomes/100 nm PS particles (two side outlets) and large EVs/500 nm PS particles (single center outlet), respectively. The entire microchannel has a uniform height $(H)$ of 50 $\mu \mathrm{m}$. The microchannels were fabricated using the standard softlithography techniques with SU8-2050 master mold on a silicon substrate. The degassed PDMS was cast over the mold and then baked in an oven at $80{ }^{\circ} \mathrm{C}$ for $2 \mathrm{~h}$. The PDMS slab was then treated with oxygen plasma and bonded to a glass substrate $(25 \mathrm{~mm} \times 75 \mathrm{~mm})$. The assembled device was placed into an oven at $70{ }^{\circ} \mathrm{C}$ for $30 \mathrm{~min}$ to enhance bonding. More detailed fabrication procedures can be found in our pervious publication. ${ }^{46,47}$ 
Particle Sample Preparation. The PEO solutions were prepared by dissolving PEO $\left(M_{\mathrm{w}}=0.6 \times 10^{6} \mathrm{Da}\right.$, Sigma-Aldrich, USA $)$ powder in $1 \times$ PBS (Sigma-Aldrich, USA) at concentrations of $0.025,0.05$, $0.075,0.1$, and $0.15 \mathrm{wt} \%$. The dissolution of PEO powder was accelerated by $1 \mathrm{~h}$ of gentle stirring (at $<30 \mathrm{rpm}$ ) and then prepared by swinging gently for $24 \mathrm{~h}$ to secure good solutions. In this work, three different fluorescent PS particles were used: 1000, 500, and $100 \mathrm{~nm}$ in diameter from Thermo Fisher Scientific, USA. The particles of 1000 and $500 \mathrm{~nm}$ were stained using Fluorescent Red (ex/em: 542/612 $\mathrm{nm}$ ) and were prepared at a concentration of $0.01 \mathrm{w} / \mathrm{v} \%$. The $100 \mathrm{~nm}$ particles were stained using Fluorescent Green (ex/em: 468/508 nm) at concentrations of $0.0002,0.002$, and $0.02 \mathrm{w} / \mathrm{v} \%$.

EV Sample Preparation from Cell Culture Medium. EVs were isolated from cell culture medium of adenocarcinomic human alveolar basal epithelial cells (A549). A549 cells were cultured in high-glucose Dulbecco's modified Eagle's medium (DMEM) supplemented with $10 \%$ exosome-free FBS (Wisent, Toronto, Canada) and $1 \%$ penicillin/ streptomycin (Wisent, Toronto, Canada) in a $5 \% \mathrm{CO}_{2}, 37{ }^{\circ} \mathrm{C}$ incubator (Thermo Scientific, USA). The cell culture supernatant containing EVs was harvested after $48 \mathrm{~h}$ of culture to reach $70 \%$ cell confluence. The EVs were isolated based on the differential centrifugation at $4{ }^{\circ} \mathrm{C}$. The collected supernatant $(300 \mathrm{~mL})$ was first centrifuged at $500 \mathrm{~g}$ for $10 \mathrm{~min}$, followed by an additional centrifugation at $2000 \mathrm{~g}$ for $20 \mathrm{~min}$ to remove cells. The treated medium was processed by membrane filtration (pore size: $0.8 \mu \mathrm{m}$, Millipore, USA), followed by centrifugation at $10000 \mathrm{~g}$ for $90 \mathrm{~min}$. Large EVs (200 to $1000 \mathrm{~nm}$ ) were then obtained by resuspending the centrifugation pellets in $500 \mu \mathrm{L}$ of PBS. To further obtain the exosomes (30 to $200 \mathrm{~nm}$ ), the remaining supernatant was concentrated to a volume of $40 \mathrm{~mL}$ by centrifugal filtration $(50 \mathrm{kDa}$ centrifugal filters, Millipore, USA) at $3000 \mathrm{~g}$ for $15 \mathrm{~min}$. The concentrated medium was then processed by membrane filtration (pore size: $0.2 \mu \mathrm{m}$, Millipore, USA), followed by ultracentrifugation at $100000 \mathrm{~g}$ for $90 \mathrm{~min}$. The exosomes were obtained by resuspending the pellet in $200 \mu \mathrm{L}$ of PBS. Before the ultracentrifugation, the medium was packaged into two ultracentrifuge tubes, and the weight difference between the two tubes was smaller than $0.05 \mathrm{~g}$. For visualization of EVs under confocal laser scanning microscopy, exosomes and large EVs were separately stained with fluorescent green and red dyes (PKH26 and PKH67, Sigma-Aldrich, USA).

Experimental Procedure. Sample fluid and sheath fluid that contain a small amount of PEO were injected into the microchannel using a syringe pump (Pump 11 Elite, Harvard Apparatus, USA) at various constant flow rates. The separation of a 500/100 nm PS particle mixture and EVs from cell culture medium was conducted at the same conditions of $c=0.1$ wt \% and flow rates of 1200 and 200 $\mu \mathrm{L} / \mathrm{h}$ for the sheath and sample fluids, respectively. The fluorescent images were obtained using an inverted microscope (Eclipse $\mathrm{Ti}$, Nikon, Japan) that was equipped with an area scan camera (piA1600$35 \mathrm{gc}$, Balser Inc., Germany) at a uniform exposure time of $4 \mathrm{~s}$. The fluorescent PS particles of 1000 and $500 \mathrm{~nm}$ and exosomes were excited by $532 \mathrm{~nm}$ green light, while $100 \mathrm{~nm}$ particles and large EVs were excited by $480 \mathrm{~nm}$ blue light. The lateral distribution of particles at each investigated downstream position was obtained by analyzing the stacked image from 200 to 400 time-elapsed fluorescent images using $z$-projection with the "average intensity" option by the Image J software package $(\mathrm{NIH})$.

Nanoparticle Tracking Analysis. To determine the recovery rate and purity of the separation, the size distributions of the initial and processed samples were measured by NTA (NanoSightNS500, Malvern Instruments, England). All the samples were diluted in PBS over concentrations between $2 \times 10^{8}$ and $1 \times 10^{9}$ particles $/ \mathrm{mL}$ to achieve the best accuracy. The size distribution data were captured and analyzed with the NTA 2.2 Analytical Software Suite. The measurements were conducted at $20{ }^{\circ} \mathrm{C}$.

Atomic Force Microscopy. The separation of exosomes was further confirmed by AFM images that were obtained at room temperature $(298 \mathrm{~K})$ using an atomic force microscope equipped with $\mathrm{SiN}$ tips with a spring constant of $0.35 \mathrm{~N} / \mathrm{m}$. The samples were prepared by applying a drop of the original or the processed sample
(10× dilution using PBS) onto mica, incubating for $10 \mathrm{~min}$, and air drying. The samples were then washed using a drop of deionized water (Milli-Q, Millipore Corp., Bedford, MA, USA) to avoid salt crystals. Images were processed and analyzed by using NanoScope Analysis.

Transmission Electron Microscopy (TEM). Exosomes collected from the side outlets were resuspended in PBS and dropped $(10 \mu \mathrm{L})$ onto electron microscope grids that had been cleaned by plasma treatment. After fixing with $2 \%$ paraformaldehyde for $20 \mathrm{~min}$ at room temperature, the grids were transferred to a $50 \mu \mathrm{L}$ drop of $1 \%$ glutaraldehyde for $5 \mathrm{~min}$ and to a $50 \mu \mathrm{L}$ drop of methyl cellulose-UA for $10 \mathrm{~min}$ on ice. After drying for $10 \mathrm{~min}$, exosomes were stained with $1 \%$ uranyl acetate for $30 \mathrm{~s}$. The dried grids were examined using an HT7700 (Hitachi, Japan) transmission electron microscope at $80 \mathrm{kV}$.

PEO Solution Properties. The relaxation times for the PEO solutions of $M_{\mathrm{w}}=600 \mathrm{kDa}$ at various concentrations are determined by the empirical formula based on capillary breakup extension rheometry measurement: $\lambda=18 \lambda_{\mathrm{Z}}\left(c / c^{*}\right)^{0.65},{ }^{37}$ where the overlapping concentration $c^{*}$ is expressed as $0.77 /[\eta]$, the intrinsic viscosity $[\eta]$ is given by the Mark-Houwink relation, $[\eta]=0.072 M_{\mathrm{w}}^{0.65}, 38$ and the Zimm relaxation time $\lambda_{\mathrm{Z}}$ is determined as $\lambda_{\mathrm{Z}}=F[\eta] M_{\mathrm{w}} \eta_{\mathrm{s}} / N_{\mathrm{A}} k_{\mathrm{B}} T$ according to Zimm's theory. Here the prefactor $F$ is 0.463 for PEO solutions, the solvent viscosity $\eta_{\mathrm{s}}$ is $1 \times 10^{-3} \mathrm{~Pa} \cdot \mathrm{s}$, and $N_{\mathrm{A}}$ and $k_{\mathrm{B}}$ represent Avogadro's number and Boltzmann's constant, respectively. ${ }^{39}$ The relaxation time $\lambda$ is consequently determined as $0.128 M_{\mathrm{w}}^{2.07} \eta_{\mathrm{s}} c^{0.65} / N_{\mathrm{A}} k_{\mathrm{B}} T$. The polymeric contribution to the viscosity $\eta_{\mathrm{p}}$ of a diluted PEO solution can be calculated as $[\eta] c \eta_{\mathrm{s}},{ }^{37}$ resulting in an expression of $0.072 \eta_{s} c M_{\mathrm{w}}^{0.65}$ according to the above relations. In the present study, the dilute PEO solutions are Oldroyd-B fluids, which can be well described using the Oldroyd-B model:

$$
\mathbf{T}+\lambda_{1} \stackrel{\nabla}{\mathbf{T}}=2 \eta_{0}\left(\mathbf{D}+\lambda_{2} \stackrel{\nabla}{\mathbf{D}}\right)
$$

where $\mathbf{T}$ is the stress tensor, $\mathbf{D}$ is the deformation tensor, $\lambda_{1}$ is the relaxation time $\lambda, \lambda_{2}=\left(\eta_{s} / \eta\right) \lambda$ is the retardation time, and $\mathbf{T}$ and $\nabla$ the upper-convected time derivatives of the stress tensor and the deformation tensor:

$$
\begin{aligned}
& \stackrel{\nabla}{\mathbf{T}}=\frac{\partial}{\partial t} \mathbf{T}+\mathbf{v} \cdot \nabla \mathbf{T}-(\nabla \mathbf{v})^{T} \cdot \mathbf{T}-\mathbf{T} \cdot(\nabla \mathbf{v}) \\
& \stackrel{\nabla}{\mathbf{D}}=\frac{\partial}{\partial t} \mathbf{D}+\mathbf{v} \cdot \nabla \mathbf{D}-(\nabla \mathbf{v})^{T} \cdot \mathbf{D}-\mathbf{D} \cdot(\nabla \mathbf{v})
\end{aligned}
$$

The Oldroyd-B model provides an expression of first normal stress difference $N_{1}$ as $N_{1}=2 \eta_{\mathrm{p}} \lambda \dot{\gamma}^{2}$, ${ }^{35}$ which is used for calculating the elastic lift forces on a particle in the present study.

Determination of the Elastic Lift Coefficient. Based on the Oldroyd-B model, $N_{1}=2 \lambda \eta_{\mathrm{p}} \lambda \dot{\gamma}^{2}$, where $\eta_{\mathrm{p}}=0.072 \eta_{\mathrm{s}} \mathrm{c} M_{\mathrm{w}}{ }^{0.65}$ and $\lambda=$ $0.128 M_{\mathrm{w}}^{2.07} \eta_{\mathrm{s}} \mathrm{c}^{0.65} / N_{\mathrm{A}} k_{\mathrm{B}} T$, respectively. From the fitting data for $a=$ $500 \mathrm{~nm}, c=0.1 \mathrm{wt} \%$, and $Q=1400 \mu \mathrm{L} / \mathrm{h}$, the average shear rate $\bar{\gamma}=7$ $\times 10^{4} \mathrm{~s}^{-1}, \eta_{\mathrm{p}}=0.41 \mathrm{mPa} \cdot \mathrm{s}, \eta=1.41 \mathrm{mPa} \cdot \mathrm{s}$, and $\lambda=0.55 \mathrm{~ms}$, we have $\Phi$ $=0.35$ and thus determine $C$ as 0.0184 .

Analytical Model of Particle Migration. The particle migration is dominated by the competition of the elastic lift force and the viscous drag force. The elastic lift force acting on a particle is

$$
\mathbf{F}_{\mathrm{e}}=C a^{3} \nabla N_{1}
$$

where $C$ is the elastic lift coefficient, $a$ the particle diameter, and $N_{1}$ the first normal stress difference defined as $N_{1}=\sigma_{11}-\sigma_{22}$; here $\sigma_{11}$ and $\sigma_{22}$ are the normal stresses in the flow and velocity gradient directions, respectively. $N_{1}$ is calculated as $N_{1}=2 \eta_{\mathrm{p}} \lambda \dot{\gamma}^{2}$ using the Oldroyd-B model for the used diluted PEO solutions, ${ }^{48}$ and the shear rate $\dot{\gamma}$ is defined as $(2 \mathbf{D}: \mathbf{D})^{1 / 2}$, where $\mathbf{D}$ is the deformation rate tensor and is expressed as $\mathbf{D}=\nabla \mathbf{u} / 2+(\nabla \mathbf{u})^{T} / 2$ (u is the fluid velocity). The viscous drag force exerting on a particle is

$$
\mathbf{F}_{\mathrm{D}}=3 \pi a \eta\left(\mathbf{u}-\mathbf{v}_{\mathrm{p}}\right)
$$

where $\mathbf{u}$ and $\mathbf{V}_{\mathrm{p}}$ are fluid and particle velocity, respectively. For the high aspect ratio of 2.5 we approximate a plane Poiseuille flow, and 
thus the particle migration is predominately along the microchannel width ( $y$ direction):

$$
\begin{aligned}
& V_{\mathrm{p}}(y)=\frac{4 C a^{2} \eta_{\mathrm{p}} \lambda \dot{\gamma}}{3 \pi \eta} \frac{\partial \dot{\gamma}}{\partial y} \\
& \dot{\gamma}=4 U_{\max }\left(\frac{1}{W}-\frac{2 y}{W^{2}}\right) \\
& \frac{\partial \dot{\gamma}}{\partial y}=-\frac{8 U_{\max }}{W^{2}}
\end{aligned}
$$

We then obtain an implicit differential equation describing the particle trajectory:

$$
\frac{\mathrm{d} y}{\mathrm{~d} x}=\frac{V_{\mathrm{p}}(y)}{V_{\mathrm{p}}(x)}=\frac{-\frac{32 \mathrm{Ca}^{2} \eta_{\mathrm{p}} \lambda U_{\max }}{3 \pi \eta W^{2}}\left(\frac{1}{W}-\frac{2 y}{W^{2}}\right)}{\left(\frac{y}{W}-\frac{y^{2}}{W^{2}}\right)}
$$

With the normalization of eq 6 by the microchannel width $W$, the maximum flow velocity $U_{\max }$ and the solvent viscosity $\eta_{\mathrm{s}}$, we obtain a neat dimensionless trajectory equation, eq 1 .

Numerical Simulation of Particle Trajectories. We conducted a Lagrangian particle tracking to model the particle motion in the 3-D microchannel using the commercial computational fluid dynamics software FLUENT 6.4 (Ansys Inc.). Due to the very low particle concentrations in the present study, we assume that the presence of the particles does not significantly influence fluid properties and the flow fields. Therefore, the motions of the particles and the ambient fluid can be solved separately. The velocity profile of a steady flow of the dilute PEO solutions used in the present study (with constant viscosities) is identical to that of a Newtonian fluid in straight channels. We thus are able to obtain the flow velocity by solving the incompressible Navier-Stokes equations in the FLUENT software:

$$
\begin{gathered}
\nabla \cdot \mathbf{u}=0 \\
\rho(\mathbf{u} \cdot \nabla) \mathbf{u}=-\nabla p+\eta \nabla^{2} \mathbf{u}
\end{gathered}
$$

where $\rho$ is the fluid density and $p$ the pressure. Based on the solved flow field, particle trajectories are predicted by integrating the force balance of the particle based on a Lagrangian formulation:

$$
\begin{aligned}
\frac{\mathrm{d} \mathbf{V}_{\mathrm{p}}}{\mathrm{d} t}= & \frac{18 \eta}{\rho_{\mathrm{p}} a^{2}} \frac{C_{\mathrm{D}} R e_{\mathrm{s}}}{24}\left(\mathbf{u}-\mathbf{V}_{\mathrm{p}}\right)+\frac{\mathbf{g}\left(\rho_{\mathrm{p}}-\rho\right)}{\rho_{\mathrm{p}}}+\frac{1}{2} \frac{\rho}{\rho_{\mathrm{p}}} \frac{\mathrm{d}\left(\mathbf{u}-\mathbf{V}_{\mathrm{p}}\right)}{\mathrm{d} t} \\
& +\frac{\mathbf{F}_{\mathrm{e}}}{\frac{1}{6} \pi a^{3} \rho_{\mathrm{p}}}
\end{aligned}
$$

where $\rho_{\mathrm{p}}$ is the particle density, $C_{\mathrm{D}}$ the drag coefficient, $R e_{\mathrm{s}}$ the relative Reynolds number, $R e_{\mathrm{s}}=\rho a\left|\mathbf{u}-\mathbf{V}_{\mathrm{p}}\right| \eta$, and $\mathbf{g}$ the gravitational acceleration. On the right-hand side of the force balance equation, the first term is the viscous drag force per unit particle mass. The $C_{\mathrm{D}}$ is calculated using an analytical expression given by Morsi and Alexander: ${ }^{49}$

$$
C_{\mathrm{D}}=a_{1}+\frac{a_{2}}{R \mathrm{e}_{s}}+\frac{a_{3}}{R e_{\mathrm{s}}^{2}}
$$

where $a_{1}, a_{2}$, and $a_{3}$ are constants that apply over a wide range of $R e_{s}$. The second term is buoyant force, which can be neglected for neutrally buoyant particles. The third term is the virtual mass force arising from the acceleration of the fluid around the particle. The fourth term is the elastic lift determined by eq 4 , which can be implemented using a userdefined function in FLUENT. A no-slip boundary condition is implemented on the microchannel walls. A pressure boundary condition is applied at the inlet to match the flow rates used in the experiments, while an atmosphere pressure is applied at the outlet. More details can be found in our pervious publication. ${ }^{50,51}$

\section{ASSOCIATED CONTENT}

\section{Supporting Information}

The Supporting Information is available free of charge on the ACS Publications website at DOI: 10.1021/acsnano.7b02277.

Additional experimental and data: Details on the microchannel design; flow fields at the outlets; elastic and inertial lift forces; trajectories of 500 and $100 \mathrm{~nm}$ PS particles in DI water; distributions of $500 \mathrm{~nm}$ particles at different PEO concentrations; separation performance of the binary mixture of 100 and $1000 \mathrm{~nm}$ PS particles; distributions of $500 \mathrm{~nm}$ particles at different flow rates; separation performance at different nanoparticle concentrations; TEM image of collected exosomes (Figures S1S9); comparison of diverse techniques for exosome separation (Table S1) (PDF)

\section{AUTHOR INFORMATION}

\section{Corresponding Authors}

*E-mail: guoqing.hu@imech.ac.cn.

*E-mail: niegj@nanoctr.cn.

*E-mail: sunjs@nanoctr.cn.

\section{ORCID}

Guangjun Nie: 0000-0001-5040-9793

Jiashu Sun: 0000-0003-4255-6202

\section{Notes}

The authors declare no competing financial interest.

\section{ACKNOWLEDGMENTS}

This work was supported financially by NSFC (11572334, 91543125), the CAS Key Research Program of Frontier Sciences (QYZDB-SSW-JSC036), and the CAS Strategic Priority Research Program (XDB22040403) for G.H., NSFC (21622503, 21475028) and Youth Innovation Promotion Association CAS (2016035) for J.S., Beijing Municipal Science \& Technology Commission (Z161100000116035) for G.N., and NSFC (U1505228) for Y.D.

\section{REFERENCES}

(1) Melo, S. A.; Luecke, L. B.; Kahlert, C.; Fernandez, A. F.; Gammon, S. T.; Kaye, J.; LeBleu, V. S.; Mittendorf, E. A.; Weitz, J.; Rahbari, N.; Reissfelder, C.; Pilarsky, C.; Fraga, M. F.; Piwnica-Worms, D.; Kalluri, R. Glypican-1 Identifies Cancer Exosomes and Detects Early Pancreatic Cancer. Nature 2015, 523, 177-182.

(2) Qi, H.; Liu, C.; Long, L.; Ren, Y.; Zhang, S.; Chang, X.; Qian, X.; Jia, H.; Zhao, J.; Sun, J.; Hou, X.; Yuan, X.; Kang, C. Blood Exosomes Endowed with Magnetic and Targeting Properties for Cancer Therapy. ACS Nano 2016, 10, 3323-3333.

(3) El Andaloussi, S.; Mager, I.; Breakefield, X. O.; Wood, M. J. A. Extracellular Vesicles: Biology and Emerging Therapeutic Opportunities. Nat. Rev. Drug Discovery 2013, 12, 347-357.

(4) Buzas, E. I.; Gyorgy, B.; Nagy, G.; Falus, A.; Gay, S. Emerging Role of Extracellular Vesicles in Inflammatory Diseases. Nat. Rev. Rheumatol. 2014, 10, 356-364.

(5) Skog, J.; Wuerdinger, T.; van Rijn, S.; Meijer, D. H.; Gainche, L.; Sena-Esteves, M.; Curry, W. T., Jr.; Carter, B. S.; Krichevsky, A. M.; Breakefield, X. O. Glioblastoma Microvesicles Transport Rna and Proteins That Promote Tumour Growth and Provide Diagnostic Biomarkers. Nat. Cell Biol. 2008, 10, 1470-1476.

(6) Balaj, L.; Lessard, R.; Dai, L.; Cho, Y.-J.; Pomeroy, S. L.; Breakefield, X. O.; Skog, J. Tumour Microvesicles Contain Retrotransposon Elements and Amplified Oncogene Sequences. Nat. Commun. 2011, 2, 180. 
(7) Théry, C.; Amigorena, S.; Raposo, G.; Clayton, A. Isolation and Characterization of Exosomes from Cell Culture Supernatants and Biological Fluids. In Current Protocols in Cell Biology; John Wiley \& Sons, Inc., 2001.

(8) Witwer, K. W.; Buzas, E. I.; Bemis, L. T.; Bora, A.; Lasser, C.; Lotvall, J.; Nolte-'t Hoen, E. N.; Piper, M. G.; Sivaraman, S.; Skog, J.; Thery, C.; Wauben, M. H.; Hochberg, F. Standardization of Sample Collection, Isolation and Analysis Methods in Extracellular Vesicle Research. J. Extracell. Vesicles 2013, 2, 20360.

(9) De Toro, J.; Herschlik, L.; Waldner, C.; Mongini, C. Emerging Roles of Exosomes in Normal and Pathological Conditions: New Insights for Diagnosis and Therapeutic Applications. Front. Immunol. 2015, 6, 203.

(10) Leshansky, A. M.; Bransky, A.; Korin, N.; Dinnar, U. Tunable Nonlinear Viscoelastic "Focusing" in a Microfluidic Device. Phys. Rev. Lett. 2007, 98, 234501.

(11) Kang, K.; Lee, S. S.; Hyun, K.; Lee, S. J.; Kim, J. M. DNA-Based Highly Tunable Particle Focuser. Nat. Commun. 2013, 4, 2567.

(12) Lim, E. J.; Ober, T. J.; Edd, J. F.; Desai, S. P.; Neal, D.; Bong, K. W.; Doyle, P. S.; McKinley, G. H.; Toner, M. Inertio-Elastic Focusing of Bioparticles in Microchannels at High Throughput. Nat. Commun. 2014, 5, 4120.

(13) Liu, C.; Ding, B.; Xue, C.; Tian, Y.; Hu, G.; Sun, J. Sheathless Focusing and Separation of Diverse Nanoparticles in Viscoelastic Solutions with Minimized Shear Thinning. Anal. Chem. 2016, 88, 12547-12553.

(14) Lu, X.; Xuan, X. Continuous Microfluidic Particle Separation via Elasto-Inertial Pinched Flow Fractionation. Anal. Chem. 2015, 87, 6389-6396.

(15) Liu, C.; Xue, C.; Chen, X.; Shan, L.; Tian, Y.; Hu, G. Size-Based Separation of Particles and Cells Utilizing Viscoelastic Effects in Straight Microchannels. Anal. Chem. 2015, 87, 6041-6048.

(16) Nam, J.; Lim, H.; Kim, D.; Jung, H.; Shin, S. Continuous Separation of Microparticles in a Microfluidic Channel via the ElastoInertial Effect of Non-Newtonian Fluid. Lab Chip 2012, 12, 13471354.

(17) Yuan, D.; Zhang, J.; Sluyter, R.; Zhao, Q.; Yan, S.; Alici, G.; Li, W. Continuous Plasma Extraction under Viscoelastic Fluid in a Straight Channel with Asymmetrical Expansion-Contraction Cavity Arrays. Lab Chip 2016, 16, 3919-3928.

(18) Hatch, A. C.; Patel, A.; Beer, N. R.; Lee, A. P. Passive Droplet Sorting Using Viscoelastic Flow Focusing. Lab Chip 2013, 13, 13081315.

(19) Lee, D. J.; Brenner, H.; Youn, J. R.; Song, Y. S. Multiplex Particle Focusing via Hydrodynamic Force in Viscoelastic Fluids. Sci. Rep. 2013, 3, 3258.

(20) Zhang, J.; Yan, S.; Yuan, D.; Zhao, Q.; Tan, S. H.; Nguyen, N.T.; Li, W. A Novel Viscoelastic-Based Ferrofluid for Continuous Sheathless Microfluidic Separation of Nonmagnetic Microparticles. Lab Chip 2016, 16, 3947-3956.

(21) Yuan, D.; Zhang, J.; Yan, S.; Pan, C.; Alici, G.; Nguyen, N. T.; Li, W. H. Dean-Flow-Coupled Elasto-Inertial Three-Dimensional Particle Focusing under Viscoelastic Flow in a Straight Channel with Asymmetrical Expansion-Contraction Cavity Arrays. Biomicrofluidics 2015, 9, 044108.

(22) Lee, K.; Shao, H.; Weissleder, R.; Lee, H. Acoustic Purification of Extracellular Microvesicles. ACS Nano 2015, 9, 2321-2327.

(23) Mao, Z.; Li, P.; Wu, M.; Bachman, H.; Mesyngier, N.; Guo, X.; Liu, S.; Costanzo, F.; Huang, T. J. Enriching Nanoparticles via Acoustofluidics. ACS Nano 2017, 11, 603-612.

(24) Yeo, L. Y.; Friend, J. R. Surface Acoustic Wave Microfluidics. Annu. Rev. Fluid Mech. 2014, 46, 379-406.

(25) Davies, R. T.; Kim, J.; Jang, S. C.; Choi, E.-J.; Gho, Y. S.; Park, J. Microfluidic Filtration System to Isolate Extracellular Vesicles from Blood. Lab Chip 2012, 12, 5202-5210.

(26) Zarovni, N.; Corrado, A.; Guazzi, P.; Zocco, D.; Lari, E.; Radano, G.; Muhhina, J.; Fondelli, C.; Gavrilova, J.; Chiesi, A. Integrated Isolation and Quantitative Analysis of Exosome Shuttled
Proteins and Nucleic Acids Using Immunocapture Approaches. Methods 2015, 87, 46-58.

(27) Murray, C.; Pao, E.; Tseng, P.; Aftab, S.; Kulkarni, R.; Rettig, M.; Di Carlo, D. Quantitative Magnetic Separation of Particles and Cells Using Gradient Magnetic Ratcheting. Small 2016, 12, 18911899.

(28) Liga, A.; Vliegenthart, A. D. B.; Oosthuyzen, W.; Dear, J. W.; Kersaudy-Kerhoas, M. Exosome Isolation: A Microfluidic Road-Map. Lab Chip 2015, 15, 2388-2394.

(29) Li, P.; Kaslan, M.; Lee, S. H.; Yao, J.; Gao, Z. Progress in Exosome Isolation Techniques. Theranostics 2017, 7, 789-804.

(30) Di Carlo, D.; Edd, J.; Humphry, K.; Stone, H.; Toner, M. Particle Segregation and Dynamics in Confined Flows. Phys. Rev. Lett. 2009, 102, 094503.

(31) Amini, H.; Lee, W.; Di Carlo, D. Inertial Microfluidic Physics. Lab Chip 2014, 14, 2739-2761.

(32) Asmolov, E. S. The Inertial Lift on a Spherical Particle in a Plane Poiseuille Flow at Large Channel Reynolds Number. J. Fluid Mech. 1999, 381, 63-87.

(33) Di Carlo, D.; Irimia, D.; Tompkins, R. G.; Toner, M. Continuous Inertial Focusing, Ordering, and Separation of Particles in Microchannels. Proc. Natl. Acad. Sci. U. S. A. 2007, 104, 1889218897.

(34) Bhagat, A. A. S.; Hou, H. W.; Li, L. D.; Lim, C. T.; Han, J. Pinched Flow Coupled Shear-Modulated Inertial Microfluidics for High-Throughput Rare Blood Cell Separation. Lab Chip 2011, 11, 1870-1878.

(35) Bird, R. B.; Armstrong, R. C.; Hassager, O.; Curtiss, C. F. Dynamics of Polymeric Liquids Vol. 1; Wiley: New York, 1987.

(36) Schonberg, J. A.; Hinch, E. J. Inertial Migration of a Sphere in Poiseuille Flow. J. Fluid Mech. 1989, 203, 517-524.

(37) Tirtaatmadja, V.; McKinley, G. H.; Cooper-White, J. J. Drop Formation and Breakup of Low Viscosity Elastic Fluids: Effects of Molecular Weight and Concentration. Phys. Fluids 2006, 18, 043101.

(38) Rodd, L. E.; Scott, T. P.; Boger, D. V.; Cooper-White, J. J.; McKinley, G. H. The Inertio-Elastic Planar Entry Flow of LowViscosity Elastic Fluids in Micro-Fabricated Geometries. J. NonNewtonian Fluid Mech. 2005, 129, 1-22.

(39) Doi, M.; Edwards, S. F. The Theory of Polymer Dynamics; Clarendon Press: Oxford, 1986.

(40) Woo, H.-K.; Sunkara, V.; Park, J.; Kim, T.-H.; Han, J.-R.; Kim, C.-J.; Choi, H.-I.; Kim, Y.-K.; Cho, Y.-K. Exodisc for Rapid, SizeSelective, and Efficient Isolation and Analysis of Nanoscale Extracellular Vesicles from Biological Samples. ACS Nano 2017, 11, $1360-1370$

(41) Hannafon, B.; Ding, W.-Q. Intercellular Communication by Exosome-Derived Micrornas in Cancer. Int. J. Mol. Sci. 2013, 14, 14240.

(42) Lamparski, H. G.; Metha-Damani, A.; Yao, J.-Y.; Patel, S.; Hsu, D.-H.; Ruegg, C.; Le Pecq, J.-B. Production and Characterization of Clinical Grade Exosomes Derived from Dendritic Cells. J. Immunol. Methods 2002, 270, 211-226.

(43) Wunsch, B. H.; Smith, J. T.; Gifford, S. M.; Wang, C.; Brink, M.; Bruce, R. L.; Austin, R. H.; Stolovitzky, G.; Astier, Y. Nanoscale Lateral Displacement Arrays for the Separation of Exosomes and Colloids Down to $20 \mathrm{Nm}$. Nat. Nanotechnol. 2016, 11, 936-940.

(44) Hur, S. C.; Tse, H. T. K.; Di Carlo, D. Sheathless Inertial Cell Ordering for Extreme Throughput Flow Cytometry. Lab Chip 2010, 10, 274-280.

(45) Chen, Y.; Wu, M.; Ren, L.; Liu, J.; Whitley, P. H.; Wang, L.; Huang, T. J. High-Throughput Acoustic Separation of Platelets from Whole Blood. Lab Chip 2016, 16, 3466-3472.

(46) Sun, J. S.; Liu, C.; Li, M. M.; Wang, J. D.; Xianyu, Y. L.; Hu, G. Q.; Jiang, X. Y. Size-Based Hydrodynamic Rare Tumor Cell Separation in Curved Microfluidic Channels. Biomicrofluidics 2013, 7, 011802.

(47) Liu, C.; Hu, G.; Jiang, X.; Sun, J. Inertial Focusing of Spherical Particles in Rectangular Microchannels over a Wide Range of Reynolds Numbers. Lab Chip 2015, 15, 1168-1177. 
(48) James, D. F. Boger Fluids. Annu. Rev. Fluid Mech. 2009, 41, $129-142$.

(49) Morsi, S. A.; Alexander, A. J. An Investigation of Particle Trajectories in Two-Phase Flow Systems. J. Fluid Mech. 1972, 55, 193-208.

(50) Gholizadeh, S.; Shehata Draz, M.; Zarghooni, M.; SanatiNezhad, A.; Ghavami, S.; Shafiee, H.; Akbari, M. Microfluidic Approaches for Isolation, Detection, and Characterization of Extracellular Vesicles: Current Status and Future Directions. Biosens. Bioelectron. 2017, 91, 588-605.

(51) Liu, C.; Xue, C.; Sun, J.; Hu, G. A Generalized Formula for Inertial Lift on a Sphere in Microchannels. Lab Chip 2016, 16, 884892. 